\title{
Investigation of slice thickness for FIB tomography in a plasma focused ion beam system
}

\author{
Brandon Van Leer ${ }^{1}$, Ron Kelley ${ }^{1}$, Bartlomiej Winiarski ${ }^{2}$ \\ ${ }^{1}$ Thermo Fisher Scientific, 5350 NW Dawson Creek Drive, Hillsboro, OR 97124 USA \\ 2 Thermo Fisher Scientific, Vlastimila Pecha 1282/12, 62700 Brno, Czech Republic
}

DualBeam FIB-SEM instrumentation has changed how researchers characterize the microstructure of materials by offering site specific analysis for S/TEM sample preparation and serial section tomography (SST) by FIB. Recent instrumentation using plasma FIB (PFIB) technology and $\mathrm{Xe}^{+}$ions offer increased milling rates because of its ability to deliver up $30-40$ times more current compared to $\mathrm{Ga}^{+}$ FIBs. This instrumentation has expanded the DualBeam's capability to characterize in 3D a variety of large grain and hard materials by automated FIB tomography [1]. Accurate slice thickness determination remains of interest in the research community for both small and large volumes.

Obtaining a 3D reconstruction by FIB tomography involves acquisition of multiple secondary or back scattered electron images of a material as slices of the material are sequentially milled through a region of interest [2]. During the reconstruction process, corrections like slice alignment and shading removal must be considered to have a successful result. There are a variety of mathematical algorithms available to for alignment in the $x-y$ plane of each image, but corrections for the z-direction must be determined on the actual slice thickness [3]. Methods exist to characterize the slice thickness variation by generating artificial reference structures in the region of interest [3,4].

Using a silicon MetroCal specimen designed to calibrate automated metrology tools, an etched square was rotated so that one corner of the etch feature was centered in the SEM image (Figure 1) [5]. To characterize slice thickness variation, automated FIB tomography experiments for a $100 \mathrm{~nm}$ and $5 \mathrm{~nm}$ slice thickness were performed using Auto Slice\&View 4. The difference in inter-distance of the two features shown in Figure 2 between 2 successive SEM images were measured. The number of pixels measured was multiplied by the size of the $\mathrm{x}$-direction of the pixel to determine the inter-slice distance (slice thickness). For small slice thickness determination the experiment employed a $30 \mathrm{keV}, 300 \mathrm{pA}$ probe. For large slice thickness $(100 \mathrm{~nm})$, a $30 \mathrm{kV}, 59 \mathrm{nA}$ probe was used. Both experiments were performed on a Thermo Scientific Helios G4 PFIB DualBeam. In the $5 \mathrm{~nm}$ FIB tomography experiment of 200 slices, results reveal an average slice thickness of $5.4 \mathrm{~nm}$ with a standard deviation of $1.2 \mathrm{~nm}$ over 50 slices. The $100 \mathrm{~nm}$ FIB tomography experiment revealed an average slice thickness of $103 \mathrm{~nm}$ with a standard deviation of $7 \mathrm{~nm}$ over 55 slices.

\section{References:}

[1] T.L. Burnett et al., Ultramicroscopy, 161, 119-129 (2016).

[2] L. Holzer et al., J. Am Ceram. Soc. 89 [8] 2577-2585 (2006).

[3] H.G. Jones et al., Ultramicroscopy, 139, 20-28 (2014).

[4] H.G. Jones et al., $2^{\text {nd }}$ International Congress on 3D Mat. Sci., 119-124 (2014).

[5] MetroBoost, www.metroboost.com. 

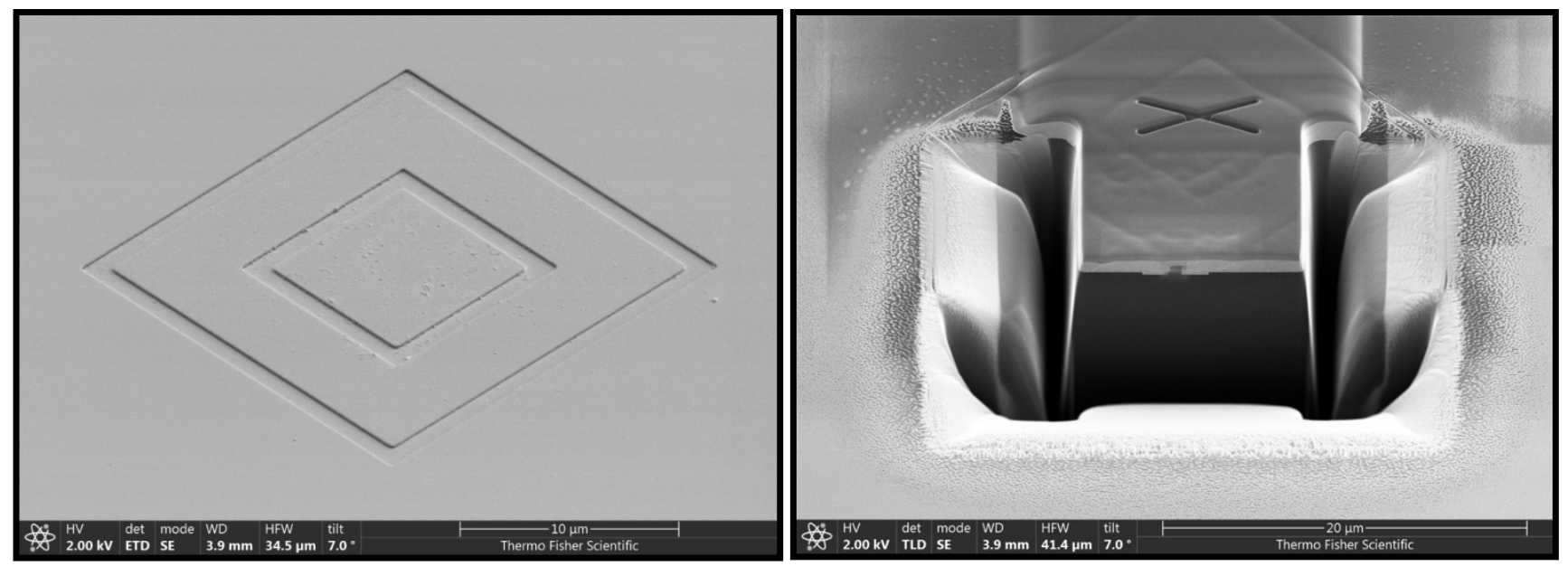

Figure 1. a) $2 \mathrm{keV} \mathrm{SEM} \mathrm{image} \mathrm{of} \mathrm{MetroCal} \mathrm{etched} \mathrm{square} \mathrm{feature;} \mathrm{b)} 2 \mathrm{keV}$ SEM image of FIB prepared cross-section site for PFIB tomography.

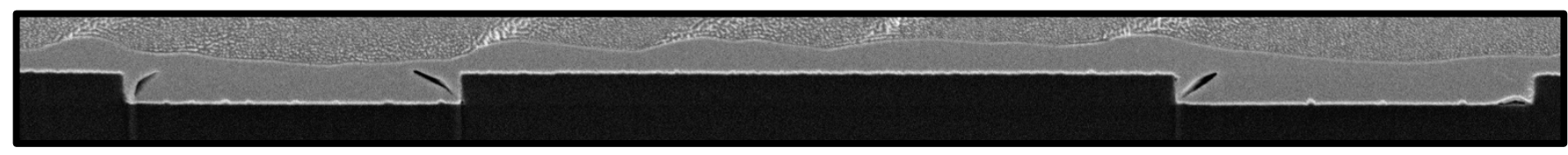

Figure 2. $2 \mathrm{keV}$ SEM cross-sectional image of MetroCal etched square feature. HFW is $6.38 \mu \mathrm{m}$, while the image resolution is $1 \mathrm{~nm} /$ pixel.

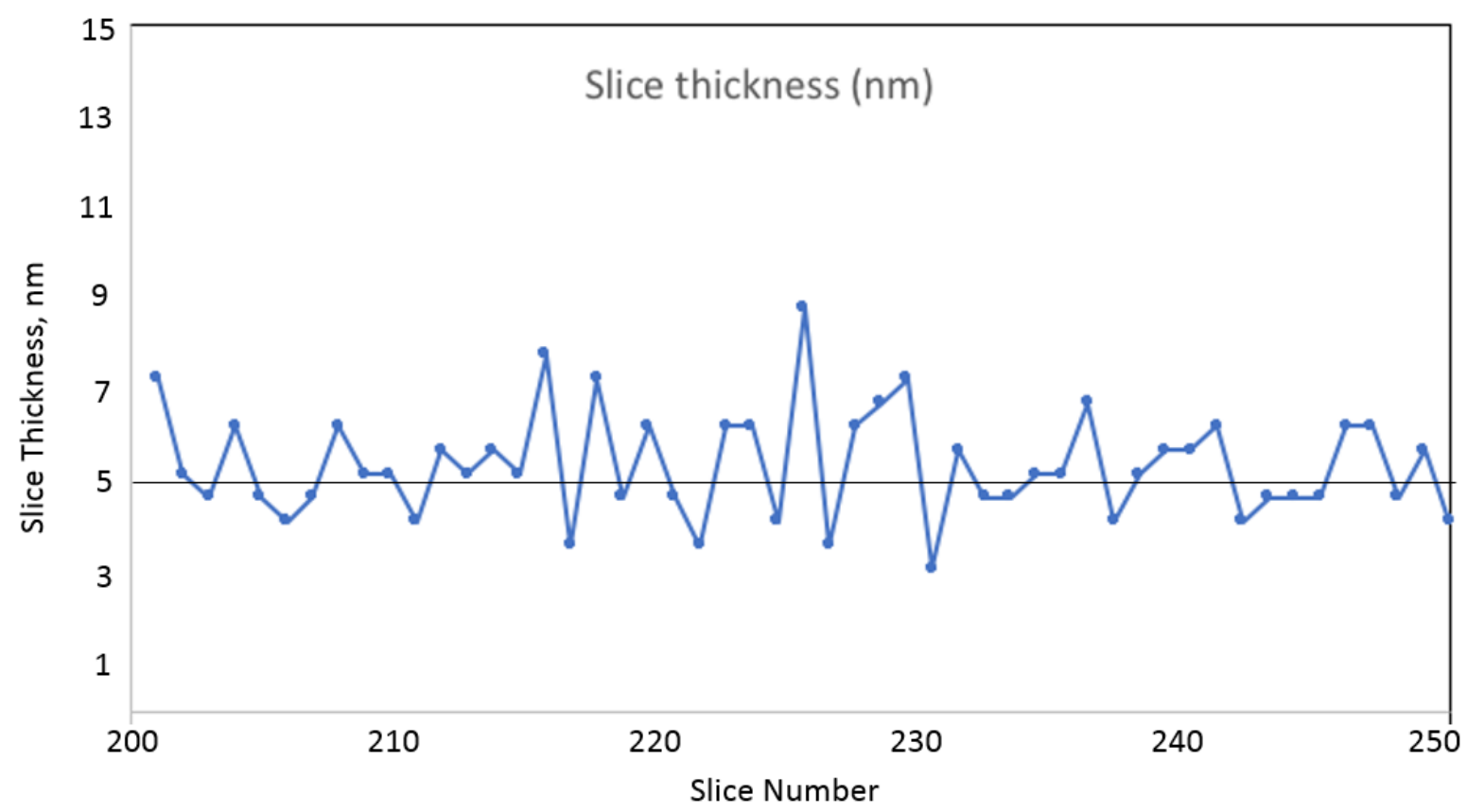

Chart 1. Summary of slice thickness measurement for 50 slices from a 200 -slice acquisition 